\title{
Assessment of tobacco control advocacy behavioural capacity among students at schools of public health in China
}

\author{
Tingzhong Yang, ${ }^{1}$ Abu S Abdullah, ${ }^{2,3}$ lan $\mathrm{R} \mathrm{H}$ Rockett, ${ }^{4} \mathrm{Mu} \mathrm{Li},{ }^{1,5}$ Yuhua Zhou, \\ Jun Ma, ${ }^{7}$ Huaping $\mathrm{Ji}^{8}{ }^{8}$ Jianzhong Zheng, ${ }^{9}$ Yuhong Zhang, ${ }^{10}$ Liming Wang ${ }^{11}$
}

${ }^{1}$ Center for Tobacco Control Research, Zhejiang University School of Medicine, Hangzhou Zhejiang, China

${ }^{2}$ Department of International Health, Boston University School of Public Health, Boston, Massachusetts, USA

${ }^{3}$ School of Public Health and Primary Care, The Chinese University of Hong Kong, Hong Kong

${ }^{4}$ Injury Control Research Center and Department of Community Medicine, West Virginia University, Morgantown, West Virginia, USA

${ }^{5}$ School of Public Health, University of Sydney, Sydney, New South Wales, Australia

${ }^{6}$ School of Public Health, Guangdong Pharmaceutical College, Guangzhou, Guangdong. China

${ }^{7}$ School of Public Health, Beijing University, Beijing, China

${ }^{8}$ School of Public Health, Nanjing Medical University, Nanjing, Jiangsu, China

${ }^{9}$ School of Public Health, Shanxi Medical University, Taiyuan,

Shanxi, China

${ }^{10}$ School of Public Health, Ningxia Medical University, Yinchuan, Ningxia, China

${ }^{11}$ School of Public Health, Haerbin Medical University, Haerbin, Helongjiang, China

\section{Correspondence to}

T Yang, School of Medicine Zijinggang Campus of Zhejiang University, Hangzhou 310058, China:

ytingzhongyang@yahoo.com

Received 6 March 2010 Accepted 14 June 2010 Published Online First 21 September 2010

\section{UNLOCKAD}

This paper is freely available online under the BMJ Journals unlocked scheme, see http:// tobaccocontrol.bmi.com/site/ about/unlocked.xhtml

\section{ABSTRACT}

Objectives To evaluate student tobacco control advocacy behavioural capacity using longitudinal trace data.

Methods A tobacco control advocacy curriculum was developed and implemented at schools of public health (SPH) or departments of public health in seven universities in China. Participants comprised undergraduate students studying the public health curriculum in these 13 Universities. A standardised assessment tool was used to evaluate their tobacco control advocacy behavioural capacity. Repeated measures analysis of variance, paired t tests and paired $\chi^{2}$ tests were used to determine differences between dependent variables across time. Multivariate analysis of variance (MANOVA) and multivariate logistic regression were used to assess treatment effects between intervention and control sites.

Results Respective totals of 426 students in the intervention group and 338 in the control group were available for the evaluation. Approximately $90 \%$ of respondents were aged 21 years or older and $56 \%$ were women. Findings show that the capacity building program significantly improved public health student advocacy behavioural capacity, including advocacy attitude, interest, motivation and anti-secondhand smoke behaviours. The curriculum did not impact student smoking behaviour.

Conclusions This study provides sufficient evidence to support the implementation of tobacco control advocacy training at Chinese schools of public health.

\section{BACKGROUND}

Tobacco smoking is the single greatest cause of preventable premature death worldwide. Almost one-third of the global adult population currently smokes and tobacco use is burgeoning in many parts of the less developed world, especially China. ${ }^{1}$ China leads the world in tobacco consumption and smoking-related deaths. Large-scale epidemiological studies have shown that smoking accounted for approximately 0.6 million deaths annually in China during the 1990s. ${ }^{2}$ This number will rise to 0.8 million in 2000, and reach 2 million per year by 2025 and 3 million by 2050. If the current smoking rate persists, approximately 100 million Chinese will die from smoking-related causes over the next 50 years. $^{3} 4$ To counter the spread of tobacco use and curb consumption worldwide, the WHO established the Framework Convention on Tobacco Control (FCTC) in 1999, which was fully endorsed by member states on 21 May 2003. The Chinese National People's Congress ratified the FCTC on 27 August 2005. However, lack of public health personnel, trained as tobacco control advocates, impedes the implementation of tobacco control activities associated with the FCTC.

There is a need to develop tobacco control curricula to use in less developed countries. ${ }^{5}$ In more developed countries, a wide variety of tobacco control curricula or training programs have been designed for medical students ${ }^{6-9}$ and other health professional students. ${ }^{10} 11$ Programs were also developed to train the tobacco control community $^{12}$ and building institutional capacity $^{13}$ on training and research on tobacco control. However, similar programs are uncommon in developing countries. ${ }^{5} 1415$ Published scientific studies on medical student views of tobacco-related issues have explored a variety of topics. Most focused on those students pursuing careers in clinical practice $^{6-15}$ However, the tobacco control advocacy curriculum rarely involved public health students. ${ }^{16}$ Currently, 76 universities in China have either a school or department of public health. About 5000 students graduate annually with a public health degree from these institutions. A significant number of these graduates work for the Chinese Centers for Disease Control and Prevention (CDCs) at provincial or local levels, and at various governmental offices, universities and in other sectors. The absence of tobacco control advocacy in their curriculum means that many lack appropriate knowledge, attitudes and skills for advocating effective tobacco control policies. This training deficit, in turn, is caused by lack of institutional resources for supporting faculty members to work in tobacco control. Funded by the Bloomberg Global Initiative (BGI), we completed an 18-month training project in seven universities in China, which aimed to build tobacco control capacity in the public health workforce. ${ }^{13} 17$ Program implementation derived from four processes. First, to develop advocacy strategies suitable for Chinese culture, we reviewed the international literature $^{18-27}$ and interviewed experienced international tobacco control advocates. Second, we developed a training program on tobacco control advocacy. Third, we targeted public health students with a $16 \mathrm{~h}$ ( $8 \mathrm{~h}$ contact and $8 \mathrm{~h}$ non-contact), training curriculum. For contact hours, students attended class room based lectures, coursework, debate and case studies. For non-contact hours, students reviewed tobacco control literatures based 
on the guidelines provided in the class and drafted a plan for smoke-free campus policy development. Fourth, representing the practice component of this curriculum, we conducted studentinitiated smoke-free campus advocacy activities. For this study, we used longitudinal trace data to evaluate the program comprehensively based on individual and group comparisons. It should be mentioned that 'advocacy' in this paper indicates policy and public attitude changes.

\section{Conceptual framework}

Our approach for building capacity comprised two aspects: (1) ability (knowledge), which covered knowledge, skills and practice; and (2) behaviour, which covered attitudes, interest and motivation, as well as behavioural change. ${ }^{21}$ These components were generated from our earlier work among students in China ${ }^{17}$ and Hong Kong ${ }^{20}$ In this paper we focused on the evaluation of behavioural capacity which included three aspects: (i) attitudes, (ii) interest and motivation and (iii) practice. The attitudes component covered general tobacco control attitudes and public health tobacco control attitudes; the interest and motivation and practice components covered public advocacy, smoking behaviour, anti-secondhand smoking behaviours and anti-smoking susceptibility.

\section{METHODS}

\section{Study design}

We conducted an intervention study to evaluate the effectiveness of the tobacco control advocacy curriculum program.

\section{Setting}

The curriculum was implemented in the schools of public health at seven universities, our intervention sites. They were Beijing University, Harbin Medical University, Ningxia Medical University, Guangdong Pharmaceutical College, Shanxi Medical University, Nanjing Medical University and Zhejiang University. Their selection was based on their regional diversity, existing research collaboration with the primary investigator and the site investigators' willingness to introduce the tobacco control advocacy curriculum to their students. For comparative purposes, we selected as controls seven universities with a public health department or college, and in close proximity to an intervention university in the same geographical region. These institutions were Beijing Capital Medical University, Qiqihar Medical College, Baotou Medical College, Zhongshan University, Hebei Medical University, Dongnan University and Zhejiang University. Control students were a year ahead of the intervention students in their public health programs.

\section{Participants}

Study participants comprised fourth-year undergraduate students studying the public health curriculum in a 5-year program (6 universities) or fifth-year undergraduate students taking the public health curriculum in a 7-year program (one university). The number of students in each class among universities varied from 29 to 115 . To encourage participation in the tobacco control curriculum, we integrated it with core courses in health education or public health.

\section{Curriculum implementation}

The program in the intervention universities followed multiple teaching formats: lecture, problem-solving-based learning, group discussion, role playing, debate and case studies. The primary aims were to equip students with basic theories, methods and skills; promote anti-tobacco activities; and conduct advocacy activities. Each intervention university delivered the course for $8 \mathrm{~h}$ contact time (classroom) and $8 \mathrm{~h}$ non-contact time. Contact hours comprised $2 \mathrm{~h}$ for the epidemiology of smoking and FCTC issues; $4 \mathrm{~h}$ for tobacco control advocacy, policies and politics; and $2 \mathrm{~h}$ for in-class practice (ie, developing, debating and discussing plans for tobacco control advocacy). In each intervention university, there were 2-3 teachers responsible for implementing the Tobacco Control Advocacy curriculum. One, usually the local principal investigator (PI), participated in a 5-day training workshop organised by the project team and international consultants. Then, the PIs delivered the same training to other potential teachers in their own institution. Usually the local PI at each intervention university delivered the main lecture while other teachers provided teaching assistance. ${ }^{17}$

The most critical element of the curriculum was that teachers and students from each intervention site had to establish and implement a smoke-free campus policy on their own campuses. In this study smoke-free campus indicates ban on smoking in all buildings and facilities within the university campus. This involved a number of key activities, including developing themes and slogans for activities to support tobacco control and ban on smoking in the campus, communicating with high-ranking officials within the university to gain support, informing all relevant bodies about the new policy. ${ }^{17}$

During the final evaluation, 12 months after program implementation, some students were inaccessible because of offcampus field practicums. However, we ensured contacting at least half of the students enrolled in a chosen class for the final evaluation. When multiple classes in an institution received the intervention, we randomly selected students from only one class for the evaluation. In each control university, we randomly selected one class of fourth-year students who were studying public health during the same semester as intervention students.

\section{Questionnaire}

We used a standardised questionnaire to assess the tobacco control advocacy capacity of students in our intervention and control universities. For study subjects, the assessment was conducted at three timepoints: beginning (baseline), middle (at 6 months) and end (at 12 months) of the project. For control students, the assessment was only conducted at baseline and 12 months.

\section{Development and piloting of the questionnaire}

The questionnaire was developed within our conceptual framework and included questions that assessed attitude towards tobacco control, advocacy interest and motivation, advocacy behaviours and tobacco use. Questions for each domain were selected by the investigators. Five experts reviewed the preliminary draft of the questionnaire for content and clarity: a tobacco control specialist, health educator, public health specialist, sociologist and psychologist. After their comments were incorporated in the questionnaire as appropriate, the survey was pilot tested with 16 participants to obtain logistical information on administration and to improve face validity. Pilot test participants were asked to provide feedback on format, content, language and completion time. Their comments were also considered for the finalisation of the questionnaire. The final instrument consisted of 52 items and required approximately $20 \mathrm{~min}$ to complete. In order to ensure greater data reliability, the survey was anonymous and all participants were encouraged to answer as honestly as possible. The Ethics Committee of each participating university approved the study protocol. 


\section{Measures}

The questionnaire covered the following information.

\section{Demographics characteristics}

Demographic information sought included age, gender, race, region of origin, educational attainment and occupations of father and mother, and monthly spending.

\section{Behavioural capacity}

Behavioural capacity included three major components, as outlined below.

\section{Attitudes}

General tobacco control attitudes: respondents were asked to rate certain tobacco control measures from the FCTC, which comprised (1) banning smoking in public places, (2) raising cigarette taxes, (3) health warnings and (4) banning tobacco advertising. They rated each item on a five-point Likert-type scale, which ranged from 1 (strongly disapprove) to 5 (strongly approve). ${ }^{17} 18$

Public health tobacco control attitudes: respondents were asked to rate their degree of approval of (1) smoke-free campus buildings in medical universities or colleges, (2) public health staff setting a tobacco control example for other members of society and (3) banning smoking among medical students and public health students. Following a five-point Likert-type scale, responses ranged from 1 (strongly disapprove) to 5 (strongly approve). ${ }^{18} 19$

\section{Advocacy interest and motivation}

Respondents were asked (1) 'are you interested in tobacco control activities?' (options were: not interested, somehow interested and very much interested), (2) 'do you want to conduct tobacco control activities as a volunteer?' (options were: don't want, somewhat want and want very much), (3) 'would you like to work to promote tobacco control?' (options were: little, medium and strong intention), (4) 'if you were in a disease control profession would you like to emphasise tobacco control?' (options were: little, medium and strong intention).

\section{Practice}

Public advocacy: this was assessed separately for family members (ie, cohabiting members in the same household) and relatives or friends. Regarding family members, we asked (1) 'do any of your family members smoke cigarettes?' (yes/no), (2) 'have you advocated to them about smoking issues in the past 3 months?' (yes/no). Regarding relatives and friends, we asked (1) 'do any of your friends and relatives smoke cigarettes?' (yes/no), (2) 'have you advocated to them about smoking issues in the past 3 months?' (yes/no).

Student current tobacco smoking: a smoker was defined as someone who smoked at the time of the survey and included daily smokers and occasional smokers. ${ }^{19} 24$

Anti-secondhand smoking behaviours: Here, respondents were asked (1) 'do you care about someone smoking around you?' (options were: very much/somewhat/not at all), (2) "what measures have been taken concerning smoking in your household?' (options were: restriction in all places/restriction in some places/no restriction), (3) 'what measures have been taken concerning smoking in your office or dormitory?' (options were: universal restriction/restriction in some places/no restriction). ${ }^{16}$

Anti-smoking susceptibility: respondents were asked (1) 'would you smoke in the future?', (2) 'would you smoke if you worked in a job with social contact which required that you smoke?', (3) 'would you smoke in the future if you worked in a disease control job?' and (4) 'would you smoke in the future if many of your friends smoked?'. Each item was rated on a fivepoint Likert-type scale ranging from 1 (least likely) to 5 (most likely).

\section{Data analysis}

We used Microsoft Excel (Microsoft, Redmond, Washington, USA) to enter all survey data into a database. Each individual in our longitudinal dataset was matched by corresponding demographic characteristics to form repeat measurement data. Most participants could be singularly identified according to birthdates; where a shared birthdate among classmates occurred, matching was accomplished using other demographics, for example, gender and region of origin.

We imported the dataset into SAS (V6.12; SAS, Cary, North Carolina, USA) for the statistical analysis. We performed our analysis in two steps. First, we conducted repeated measures analysis of variance, paired tests and paired $\chi^{2}$ tests to determine if there were differences in dependent variables across time, in order to assess the effect of the repeated measurement factor and time. We used the GIM program for repeated measures analysis of variance to analyse the continuous dependent variables and CATMODE to analyse the categorical dependent variables. Multiple comparisons were performed to evaluate significant differences across time. Second, to evaluate differences in final and baseline measures of the dependent variables between intervention and control groups, that is, to assess intervention effectiveness, we used multivariate analysis of variance (MANOVA) for continuous variables and multivariate logistic regression analysis for categorical variables. All statistical significance testing was two sided, using a conventional 5\% cut-off.

\section{RESULTS}

Linkable baseline, midpoint and final assessment data were available for analysis on 426 students in the intervention group $(n=463)$ and were included in the analyses. For the student control group $(n=356)$, linkable baseline and final assessment data were available on 338 students. The response rate was $92.0 \%$ in the intervention group and $94.4 \%$ in the control group.

\section{Demographic characteristics of respondents}

The majority $(90 \%)$ of respondents were aged 21 years or older and $44 \%$ were men (table 1). Students in the intervention group were different than those in the control group in terms of age, race, region of origin, parental education and maternal occupation (table 1).

\section{Questionnaire reliability}

Cronbach $\alpha$ values by domain were 0.589 for general tobacco control attitudes, 0.784 for public health tobacco control attitudes, 0.723 for advocacy interest and motivation, 0.653 for antisecondhand smoking behaviours and 0.882 for anti-smoking susceptibility. These values suggest that the measures used in the questionnaires had acceptable reliability.

The results shown in table 2 suggest that student behavioural capacity, except smoking behaviour, significantly improved with increased intervention time; but they did not vary by time across variables, except for general tobacco control attitudes and anti-secondhand smoking behaviours, in the control group.

\section{Treatment effects (intervention vs control groups)}

Tables 3 and 4 shows that attitudes, advocacy interest and motivation variables, and most of the practice variables had significantly changed after the intervention. However, there was 
Table 1 Demographic characteristics of the sample

\begin{tabular}{|c|c|c|c|c|c|}
\hline Group & n (\%) & $\begin{array}{l}\text { Intervention } \\
\text { group } \\
\text { n (\%) }\end{array}$ & $\begin{array}{l}\text { Control } \\
\text { group } \\
\text { n (\%) }\end{array}$ & $\chi^{2}$ & $\begin{array}{l}p \\
\text { Value }\end{array}$ \\
\hline \multicolumn{6}{|l|}{ Age in years } \\
\hline 21 & $77(10.1)$ & $17(4.0)$ & $60(17.8)$ & \multirow{5}{*}{220.00} & \multirow[t]{5}{*}{$\mathrm{p}<0.01$} \\
\hline 21 & $174(22.8)$ & $42(9.9)$ & $132(39.1)$ & & \\
\hline 22 & $188(24.6)$ & $93(21.8)$ & $95(28.1)$ & & \\
\hline 23 & $195(25.5)$ & $156(36.6)$ & 39 (11.5) & & \\
\hline $24+$ & $130(17.0)$ & $118(27.7)$ & $12(3.6)$ & & \\
\hline \multicolumn{6}{|l|}{ Gender } \\
\hline Male & $339(44.4)$ & $191(44.8)$ & $148(43.8)$ & \multirow[t]{2}{*}{0.78} & \multirow[t]{2}{*}{$p>0.05$} \\
\hline Female & $425(55.6)$ & $235(55.2)$ & $190(56.2)$ & & \\
\hline \multicolumn{6}{|l|}{ Race } \\
\hline Han & 714 (93.5) & $391(91.8)$ & $323(95.6)$ & \multirow[t]{2}{*}{4.40} & \multirow[t]{2}{*}{$\mathrm{p}<0.05$} \\
\hline Other & $50(6.5)$ & $35(8.2)$ & $15(4.4)$ & & \\
\hline \multicolumn{6}{|l|}{ Region of origin } \\
\hline Northeast & $90(11.8)$ & $32(7.5)$ & $58(17.2)$ & \multirow[t]{7}{*}{72.17} & \multirow[t]{7}{*}{$\mathrm{p}<0.01$} \\
\hline North & $122(16.0)$ & $63(14.8)$ & $59(17.5)$ & & \\
\hline Northwest & $86(11.3)$ & 57 (13.4) & $29(8.6)$ & & \\
\hline East & $145(19.0)$ & $69(16.2)$ & $76(22.5)$ & & \\
\hline South & $252(33.0)$ & $184(43.2)$ & $68(20.1)$ & & \\
\hline Southwest & $26(3.4)$ & $9(2.1)$ & $7(5.0)$ & & \\
\hline Middle South & $43(5.6)$ & $12(2.8)$ & $31(9.2)$ & & \\
\hline \multicolumn{6}{|c|}{ Expenditure per month (RMB) } \\
\hline 400 & $238(31.2)$ & $71(16.7)$ & $60(17.8)$ & \multirow[t]{4}{*}{7.91} & \multirow[t]{4}{*}{$\mathrm{p}>0.05$} \\
\hline $400-599$ & $214(28.0)$ & $170(39.9)$ & $155(45.9)$ & & \\
\hline $600-799$ & $118(15.4)$ & $116(27.2)$ & $63(18.6)$ & & \\
\hline $800+$ & $194(25.4)$ & $69(16.2)$ & $60(17.8)$ & & \\
\hline \multicolumn{6}{|l|}{ Paternal education } \\
\hline Elementary school & $80(10.5)$ & $52(12.2)$ & $28(8.3)$ & \multirow[t]{4}{*}{16.56} & \multirow[t]{4}{*}{$\mathrm{p}<0.01$} \\
\hline Junior high school & $192(25.1)$ & $124(29.1)$ & $68(20.1)$ & & \\
\hline High school & $311(40.7)$ & $167(39.2)$ & $144(42.6)$ & & \\
\hline College and above & $181(23.7)$ & $83(19.5)$ & $98(29.0)$ & & \\
\hline \multicolumn{6}{|l|}{ Maternal education } \\
\hline Elementary school & $166(21.7)$ & $100(23.5)$ & $66(19.5)$ & \multirow[t]{4}{*}{11.63} & \multirow[t]{4}{*}{$p<0.01$} \\
\hline Junior high school & $225(29.5)$ & $140(32.9)$ & $85(25.1)$ & & \\
\hline High school & $261(34.2)$ & $135(31.7)$ & $126(37.3)$ & & \\
\hline College and above & $112(14.7)$ & $51(12.0)$ & $61(18.0)$ & & \\
\hline \multicolumn{6}{|l|}{ Paternal occupation } \\
\hline $\begin{array}{l}\text { Operations and } \\
\text { commercial }\end{array}$ & $520(68.1)$ & 301 (70.7) & $219(64.8)$ & \multirow[t]{3}{*}{3.14} & \multirow[t]{3}{*}{$\mathrm{p}>0.05$} \\
\hline Staff & $188(24.6)$ & $95(22.3)$ & $93(27.5)$ & & \\
\hline Technical and teaching & $56(7.3)$ & $30(7.0)$ & $26(7.7)$ & & \\
\hline Maternal occupation & & & & & \\
\hline $\begin{array}{l}\text { Operations and } \\
\text { commercial }\end{array}$ & $562(73.6)$ & $329(77.2)$ & $233(68.9)$ & 7.40 & $\mathrm{p}<0.05$ \\
\hline Staff & $133(17.4)$ & $61(14.3)$ & $72(21.3)$ & & \\
\hline Technical and teaching & $69(9.0)$ & $36(8.5)$ & $33(9.8)$ & & \\
\hline
\end{tabular}

no change in smoking behaviour. It should be mentioned that smoke-free campus policies were developed and implemented in six universities. Beijing University already had a smoke-free campus policy before the initiation of this project.

\section{DISCUSSION}

Our evaluation indicates that a tobacco control training program can significantly improve advocacy behavioural capacity of public health students, including their advocacy attitudes, interest and motivation regarding anti-secondhand smoking behaviours. To our knowledge, this is the first assessment study of a tobacco control training program in China. We believe that advocacy behavioural capacity is very important for public health students, so that they can integrate tobacco control activities into their future public health careers. How public health students' education and training will be used in practice could be guided by the degree to which they hold favourable attitudes, interests and motivation concerning tobacco control advocacy. $^{21}$

In this study students in the intervention schools were older $(p<0.01)$ than those in the control group, which might be explained by the fact that participants in one of the intervention universities were in the 7-year undergraduate curriculum and were older than those taking the 5-year curriculum.

In our intervention study, tobacco control advocacy behavioural capacity included attitudes, interests and motivation, and practices (ie, advocacy behaviours, anti-secondhand smoking behaviours and anti-smoking susceptibility). We view each of these elements as necessary for students to become competent public health professionals. Some studies showed that increased interest and motivation promoted implementation of tobacco control activities among medical workers. ${ }^{28-31}$ In our intervention study, we evaluated tobacco control advocacy behaviour in public health students from the perspective of future practice.

We found that general tobacco control attitudes and antisecondhand smoking behaviours have improved in the control group. This may be because tobacco control has a higher profile in China today than in the past, which is due to the implementation of several internationally funded projects and enhanced government initiatives raising public awareness. However, indicating program effectiveness, the intervention group showed greater improvement than the control group on most of our outcome measures.

We found that our training program did not have any impact on the smoking behaviour of public health students. This may be because we emphasised tobacco control advocacy among students, which is related to shaping anti-smoking policy and encouraging non-smoking among others rather than among themselves. We deem it is important that public health workers set examples for others as non-smokers during advocacy campaigns. Perhaps another reason why our intervention did not impact smoking behaviour was the low prevalence of smoking among our participants. Since smoking is negatively associated with initiation of tobacco control activities, ${ }^{28-30}$ future studies should confront smoking attitudes and behaviour of students directly to achieve the goal of implementing appropriate and effective smoking cessation programs across China.

In showing the effectiveness of our program, this study indicates the need to train future public health professionals in tobacco control advocacy. Tobacco smoking continues to be the most significant preventable cause of death and disability in China. If the Healthy China 2010 objectives are met, this will reflect the industriousness and due diligence of a trained public health workforce. However, to address tobacco-related health problems in particular, the government and other agencies should support academic and research-related tobacco control training opportunities in the schools of public health in order to test and develop an appropriate prevention and control model for China. Through this study, we learnt of a number of issues that need to be considered for training and building capacity in tobacco control advocacy among public health students. First, tobacco control advocacy should be embedded in the public health curriculum of each university. Second, teaching faculty should be well trained and competent to deliver effective tobacco control advocacy courses in their universities. Third, there should be more emphasis on how students use their acquired knowledge and skills in real world settings. ${ }^{8}$ This could be accomplished by incorporating case studies or mini projects as assignments. In our study, student advocacy was improved 


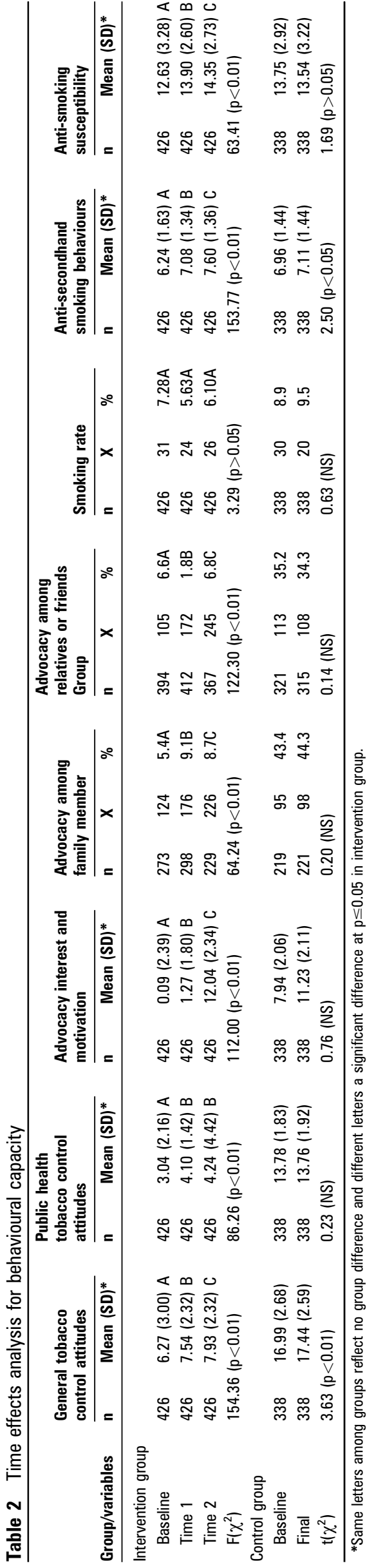

Table 3 Treatment effects analysis using multivariate analysis of variance (MANOVA) on response variables

\begin{tabular}{|c|c|c|c|c|}
\hline Variables & $\begin{array}{l}\text { Mean of difference } \\
\text { (final baseline) }\end{array}$ & $\begin{array}{l}\text { SD of difference } \\
\text { (final baseline) }\end{array}$ & $\mathbf{F}$ & p Value \\
\hline \multicolumn{5}{|c|}{ General tobacco control attitudes } \\
\hline Control group & 0.59 & 0.49 & 129.95 & 0.0001 \\
\hline Intervention group & 0.91 & 0.28 & & \\
\hline \multicolumn{5}{|c|}{ Public health tobacco control attitudes } \\
\hline Control group & 0.34 & 0.47 & 99.56 & 0.0001 \\
\hline Intervention group & 0.69 & 0.47 & & \\
\hline \multicolumn{5}{|c|}{ Advocacy interest and motivation } \\
\hline Control group & 0.58 & 0.49 & 157.26 & 0.0001 \\
\hline Intervention group & 0.93 & 0.26 & & \\
\hline \multicolumn{5}{|c|}{ Anti-secondhand smoking behaviours } \\
\hline Control group & 0.41 & 0.49 & 280.94 & 0.0001 \\
\hline Intervention group & 0.89 & 0.31 & & \\
\hline \multicolumn{5}{|c|}{ Anti-smoking susceptibility } \\
\hline Control group & 0.38 & 0.48 & 125.86 & 0.0001 \\
\hline Intervention group & 0.65 & 0.48 & & \\
\hline
\end{tabular}

through development of smoke-free campus policy advocacy activities. ${ }^{17}$ Moreover, the key issue was that students apply their tobacco control education in their future work. To assess this we contacted nine students working at different CDC offices a year after the project completion; six of them thought the tobacco control knowledge and skills acquired at the university were very useful to their work, two thought they were useful and one was uncertain. This suggests that our project was beneficial to the students' future work. We will conduct a longer-term follow-up to assess the long-term effect of our training intervention. Fourth, we suggest that standardised methods and programs be used for advocacy capacity training. They should be formed through a series of consultations and as a result of further research activities, such as in developing a culturally sensitive training program, establishing and implementing an evaluation plan, creating a teaching manual and establishing guidelines for program implementation and evaluation as we did in our study. ${ }^{17}$ In fact, our study can serve as a demonstration project to expand similar programs at more schools of public health across the country.

The tobacco epidemic is a major public health threat to many developing countries, including China. Only by means of sustained and coordinated efforts and through a comprehensive program can tobacco control be achieved. Comprehensive programs require involvement of public health professionals with specific training in tobacco control advocacy. The most effective time to implement advocacy training for public health professionals is when they are students and motivated to learn. ${ }^{32}$ The effectiveness of our program suggests that it could

Table 4 Treatment (intervention and control) effects analysis results using multivariate logistic regression model on response variables

\begin{tabular}{lcll}
\hline Variables & $\begin{array}{l}\text { Mean difference } \\
\text { (final baseline) }\end{array}$ & RR & 95\% Cl \\
\hline Advocacy among family members & & \\
$\quad$ Control group & 0.9 & 1.00 & - \\
$\quad$ Intervention group & 33.8 & 5.56 & 3.45 to 8.33 \\
Advocacy among relatives or friends & & \\
$\quad$ Control group & -0.9 & 1.00 & - \\
$\quad$ Intervention group & 35.7 & 4.55 & 2.17 to 9.09 \\
Smoking rate & & & - \\
$\quad$ Control group & 0.6 & 1.00 & - \\
Intervention group & -0.9 & 1.70 & 0.78 to 3.70 \\
\hline
\end{tabular}




\section{What this paper adds}

- The findings from our present work suggest that implementation of a tobacco control advocacy curriculum within the Schools of Public Health in China is feasible.

- The capacity building curriculum, if implemented in an organised manner, would improve public health students' tobacco control advocacy behavioural capacity (ie, advocacy attitude, interest, motivation and anti-secondhand smoke behaviours).

be further extended to students in other universities and tested with an additional research element, namely, a cessation component, to enhance effectiveness.

Our study has five limitations. First, only two assessments were conducted with the control group, compared to three with the evaluation group. Second, some outcome variables, related to smoke-free campus advocacy, were not included in our behavioural capacity questionnaire. However, we were able to gather these data from evaluations of students' class performance in tobacco advocacy practice. Third, participation of universities in the study was by non-random selection and, therefore, the findings may not be generalisable to all universities in China. Fourth, students involved in this study were at a later stage of education; they could have been exposed to more tobaccorelated knowledge, such as tobacco control movement in society and aetiology about smoking in their clinical medicine curriculum, which might have attenuated the findings of the current study. However, we think our study eliminated this limitation because we had a control group. Fifth, there was an established working relationship between the investigator and those who implemented the curriculum, which might play a positive role in the implementation process. However, we believe such a collaborative nature is common and natural, and the curriculum was implemented by the local investigators with technical support from the key personnel. Therefore we believe the collaborative nature should not have any affect on the project's effectiveness.

In conclusion, our study generated sufficient evidence to support implementation of tobacco control advocacy training in Chinese schools of public health. Our findings also support an imperative to employ standard assessment tools to implement a rigorous evaluation of such training.

Acknowledgements This study was a Bloomberg Global Initiative project supported by the International Union Against Tuberculosis and Lung Disease (U-China-1-15). We especially thank technical and financial experts from the Union and China Resource Centre for providing us with much valuable technical support. We sincerely thank Anne Jones, Yan Lin, Trish Fraser, Balasu Bramanian Gopalan and Ning Dong for their generous contribution in achieving project targets.

Funding Bloomberg Global Initiative project supported by the International Union Against Tuberculosis and Lung Disease (U-China-1-15).

\section{Competing interests None.}

\section{Patient consent Obtained.}

Ethics approval This study was conducted with the approval of the Ethics Committee of the participating Universities.

Contributors TY and ASA originated the study, developed study protocol and guided the data analysis. TY supervised all aspects of the study implementation. ML, YZ, JM, $\mathrm{HJ}, \mathrm{JZ}, \mathrm{YZ}$ and LM assisted in the study implementation and data collection in each study site. TY and ASA drafted the manuscript. IRHR provided in-depth comments in the earlier draft of the manuscript. TY, ASA and IRHR compiled all the comments and finalised the final draft of the manuscript. All authors reviewed drafts of the manuscript and approved the version to be published.
Provenance and peer review Not commissioned; externally peer reviewed.

\section{REFERENCES}

1. World Health Organization. WHO report on global tobacco epidemic 2008: MPOWER package. Geneva: WHO, 2008:7-9.

2. Liu BO, Peto R, Chen ZM, et al. Emerging tobacco hazards in China: retrospective proportional mortality study of one million deaths. BMJ 1998;17:1411-22.

3. Niu SR, Yang GH, Chen ZM, et al. Emerging tobacco hazards in China: early mortality results from a prospective study. BMJ 1998;317:1423-4.

4. Peto R, Chen ZM, Boreham J. Tobacco the growing epidemic. Nat Med 1999;5:15-17.

5. Abdullah AS, Husten CG. Promotion of smoking cessation in developing countries: a framework for urgent public health interventions. Thorax 2004;59:623-30.

6. Ferry LH, Grissino LM, Runfola PS. Tobacco dependence curricula in US undergraduate medical education. JAMA 1999;282:825-9.

7. Corelli RL, Kroon LA, Chung EP, et al. Statewide evaluation of a tobacco cessation curriculum for pharmacy students. Prev Med 2005:40:888-95.

8. Roddy E, Rubin P, Britton J. A study of smoking and smoking cessation on the curricula of UK medical schools. Tob Control 2004:13:74-7.

9. Brown RL, Pfeifer JM, Gjerde CL, et al. Teaching patient-centered tobacco intervention to first-year medical students. J Gen Intern Med 2004;19:534-9.

10. Berman BA, Kominski GF. The UCLA tobacco control program. Public Health Rep 2006;121:515-20.

11. Savage SE, Halperin AC. Building an academic tobacco control program while forging links with practice: the University of Washington Tobacco Studies Program. Public Health Rep 2006;121:529-37.

12. Stillman F, Wipfli H, Samet J. Reaching and educating the global tobacco contro community: innovative approaches to tobacco control training. Public Health Rep 2006;121:521-8

13. Warner KE, Burkley DJ. The development, achievements, and aspirations of a university network dedicated to tobacco control research and education. Public Health Rep 2006;121:509-14.

14. Daudt AW, Alberg AJ, Prola JC et al. A first step incorporating smoking education into a Brazilian medical school curriculum: results of a survey to assess the cigarette smoking knowledge, attitudes, behaviour, and clinical practices of medical students. J Addict Dis 1999:18:19-29.

15. Tao S, Croucher R, Pau A. Impact of the curriculum on Chinese dental students tobacco control attitudes and beliefs: a case study in Harbin, China. Int Dent J 2008;58:181-6.

16. Gonzales R, Glik D, Davoudi $M$, et al. Health: integrating theory, research, and practice for tobacco control. Am Behav Sci 2004:48:189-201.

17. Yang T, Yang X, Lv Q, et al. China's first historic efforts to develop a tobacco control advocacy workforce via schools of public health. Tob Control 2009;18:422-4.

18. Chapman S. Public health advocacy and tobacco control. MA, USA: Blackwell Publishing Inc, 2007

19. John R. Strategy planning for tobacco control advocacy. http://strategyguides globalink.org/advocacy.htm.

20. Abdullah AS. Clinical skills for the treatment of tobacco dependency: developmen and implementation of curriculum for medical students in Hong Kong. 11th Annual Meeting of Society for Research on Nicotine and Tobacco (SRNT), 20-23 March 2005, Prague, Czech Republic. Abstract book (Pre meeting conference): Abstract GTR-003, page 1.

21. Yang T. Smoking. In: Yang T. Health behavior theory and research. Beijing: People Health Press, 2007:30-2.

22. Abdullah AS, Rahman AM, Suen CW, et al. Investigation of Hong Kong doctors current knowledge, beliefs, attitudes, confidence and practices: Implications for the treatment of tobacco dependency. J Chin Med Assoc 2006:69:461-71.

23. Chapman S, Borland R, Lal A. Has the ban on smoking in New South Wales restaurants worked? A comparison of restaurants in Sydney and Melbourne. Med Aust 2001;174:512-15.

24. Yang T, Wu Y, Dai Abdullah AS, et al. Attitudes and behavioural response toward some tobacco control measures from the FCTC among Chinese urban residents. BMC Public Health 2007:7:248.

25. Yang T, Wu J, Rockett IR, et al. Smoking patterns among Chinese rural-urban migrant workers. Public Health 2009;123:743-9

26. Fried J, Reid B, DeVore L. A comparison of health professions student attitudes regarding tobacco curricula and interventionist roles. J Dent Educ 2004;68:370-7.

27. Jenkins K, Ahijevych K. Nursing students' belief about smoking, their own smoking behaviors, and use of professional tobacco treatment intervention. Appl Nurs Res 2003;16:164-72.

28. Sarna L, Brown JK, Lillington L, et al. Tobacco-control attitudes, advocacy, and smoking behaviors of oncology nurses. Oncol Nurs Forum 2000;27:1519-28.

29. Mcllvain HE, Backer EL, Crabtree BF. Physician attitudes and the use of office-based activities for tobacco control Family Medicine. Fam Med 34:114-19.

30. Takashi Ohida T, Sakurai H, Mochizuki Y, et al. Smoking prevalence and attitudes toward smoking among Japanese physicians. JAMA 2001;285:2643-8.

31. Bass F. Mobilizing physicians to conduct clinical intervention in tobacco use through a medical-association program: 5 years' experience in British Columbia. Can Med Assoc J 1996:154:159-64.

32. Abdullah AS, Fielding R, Hedley AJ, et al. Training medical students about HIV/AIDS. Med Educ 1999;33:472-3. 